\title{
LUT
}

University

Liquid-liquid synthesis of oximes from carbonyl compounds: formation under neutral conditions and degradation at acidic hydrometallurgical process conditions

Kabugo James, Virolainen Sami, Paatero Erkki, Sainio Tuomo

This is a Final draft version of a publication

published by John Wiley \& Sons, Inc.

in Journal of Chemical Technology and Biotechnology

DOI: $10.1002 / j c t b .5143$

Copyright of the original publication: (c) 2016 Society of Chemical Industry

Please cite the publication as follows:

Kabugo, J., Virolainen, S., Paatero, E., Sainio, T., 2016. Liquid-liquid synthesis of oximes from carbonyl compounds: Formation at neutral conditions and degradation at acidic hydrometallurgical process conditions. Journal of Chemical Technology Biotechnology, Available online. doi:10.1002/jctb.5143

This is a parallel published version of an original publication.

This version can differ from the original published article. 


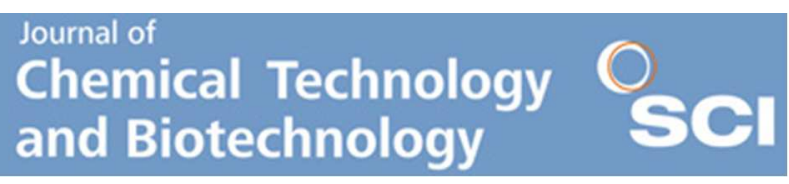

\section{Liquid-liquid synthesis of oximes from carbonyl compounds: Formation at neutral conditions and degradation at acidic hydrometallurgical process conditions}

\begin{tabular}{|r|l|}
\hline Journal: & Journal of Chemical Technology \& Biotechnology \\
\hline Manuscript ID & JCTB-16-0720.R1 \\
\hline Wiley - Manuscript type: & Research Article \\
\hline Complete List of Authors: & $\begin{array}{l}\text { Kabugo, James; Outotec (Finland) Oy, Pori Research Center } \\
\text { Virolainen, Sami; Lappeenranta University of Technology, Chemical } \\
\text { technology } \\
\text { Paatero, Erkki; Lappeenranta University of Technology, School of } \\
\text { Engineering Science } \\
\text { Sainio, Tuomo; Lappeenranta University of Technology, Chemical } \\
\text { Technology }\end{array}$ \\
\hline Key Words: & $\begin{array}{l}\text { Hydrometallurgy, Liquid-Liquid Extraction, Equilibrium, Kinetics, Modelling, } \\
\text { Reaction }\end{array}$ \\
\hline
\end{tabular}




\section{Liquid-liquid synthesis of oximes from carbonyl compounds: Formation at} neutral conditions and degradation at acidic hydrometallurgical process

\section{conditions}

9

James Kabugo $^{\text {a, b }}$, Sami Virolainen ${ }^{\left.\mathrm{a}^{*}\right)}$, Erkki Paatero ${ }^{\mathrm{a}}$ and Tuomo Sainio ${ }^{\mathrm{a}}$

13 a Lappeenranta University of Technology, Skinnarilankatu 34, FI 53850 Lappeenranta, Finland

$14{ }^{\mathrm{b}}$ Outotec (Finland) Oy, Kuparitie 10, 28330 Pori

15

16

17

Revision 1

19

20

21

22

23

24 *) Corresponding author, e-mail: Sami.Virolainen@lut.fi .Tel.: +358407093444

25 
1 ABSTRACT

2 BACKGROUND

3 In order to elucidate kinetics in the synthesis of oxime liquid-liquid extraction reagents, used as

4 ligands in hydrometallurgical copper production, the oximation reactions of salicylaldehyde and

5 2'-hydroxyacetophenone with hydroxylamine were studied in two phase system. Two

6 experimental methods were applied: CSTR reactor under vigorous mixing and batch reactor

7 under normal agitation. The reverse reaction in acidic conditions was also examined, because it

8 results hydroxyoxime reagent losses in copper liquid-liquid extraction process.

9 RESULTS

10 The overall oximation reaction was observed to be of a second order, first order with respect to 11 each reactant. The estimated activation energy for oximation of salicylaldehyde was $34.2 \mathrm{~kJ} / \mathrm{mol}$, 12 and interfacial reaction rate constant at $40^{\circ} \mathrm{C} 4.81 \cdot 10^{-3} \mathrm{~L} / \mathrm{mol} / \mathrm{s}$. Used model, including 13 empirical description for interfacial reactant concentrations, described the salicylaldehyde 14 oximation reaction well in the wide concentration range. In the batch reactor over 0.99 15 conversion for salicylaldehyde was achieved in $15 \mathrm{~min}$ at $50^{\circ} \mathrm{C}$, but for $2^{\prime}$-hydroxyacetophenone 16 maximum reaction conversion of only 0.17 was achieved at $70{ }^{\circ} \mathrm{C}$ in $60 \mathrm{~min}$. Both, aldoxime and 17 ketoxime, were hydrolyzed into their corresponding carbonyl compounds in presence of $180 \mathrm{~g} / \mathrm{L}$ 18 sulfuric acid. The ketoxime was completely converted into a ketone, but with the aldoxime 19 equilibrium was observed at $80 \%$ reagent degradation.

\section{CONCLUSION}

21 Liquid-liquid system in neutral conditions is applicable for oximation of especially aldehydes 22 generated by degradation of hydroxyoxime solvent extraction reagents. For ketones the reaction 
2

3

4

5

6

7

8

9

10

11

12

13

14

15

16

17

18

19

20

21

22

23

24

25

26

27

28

29

30

31

32

33

34

35

36

37

38

39

40

41

42

43

44

45

46

47

48

49

50

51

52

53

54

55

56

57

58

59

60

1 is much slower. The degradation reaction was shown to be significant in conditions similar to

2 copper liquid-liquid extraction stripping stage.

3 Keywords: Hydroxyoximes, aldehyde, ketone, hydroxylamine, hydrolytic degradation 


\section{INTRODUCTION}

2 Hydroxyoxime ligands, both aldoximes and ketoximes, are extensively used as extractants in

3 hydrometallurgy, for example in the recovery and purification of copper from aqueous pregnant

4 leach solutions [1]. They are also applied as mineral collectors in minerals processing [2].

5 Hydroxyoxime copper reagents are selective, and also otherwise technically very functional to

6 the separation task, but generally regarded expensive due to certain challenges in their

7 manufacturing process.

8 Synthesis of hydroxyoxime ligands from their carbonyl compounds, aldehyde or ketone, is

9 usually done with the use of hydroxylamine or its acid salts [3]. The rates of oximation reactions

10 are notably slow, and may require several hours especially under ambient conditions. This is

11 particularly true for carbonyl compounds with very low solubility in aqueous solutions, like the

12 hydroxyoximes for the copper hydrometallurgy. Several synthesis methods to increase oximation

13 rates have been proposed in literature. These include application of micellar [3-4] and phase-

14 transfer catalysis [5] in liquid-liquid systems, solid-organic systems [6] and single organic phase

15 systems [7-9].

16 The kinetics of the oximation reaction has been studied in the past for various aldehydes and

17 ketones $[3,10]$. The reaction rate tends to vary widely among different carbonyl reagents

18 because steric hindrance and electronic effects play an important role in the reactivity of

19 carbonyl compounds [10-13]. Another important factor is the reaction medium, as was observed

20 while developing analytical methods for quantification of aldehydes and ketones in 1960's [14].

21 The reverse reaction to oximation usually occurs via acid catalyzed hydrolysis. This unwanted

22 phenomenon leads to degradation of alkyl hydroxyoximes when they are employed for liquid-

23 liquid extraction of metals in the hydrometallurgical industry [15-18]. In copper liquid-liquid 
1 extraction plants, for example, sulfuric acid concentration of $180 \mathrm{~g} / \mathrm{L}$ is typical in the stripping

2 stage, which favors the hydrolytic degradation [18]. It is known that ketoximes are normally

3 more stable against the hydrolysis than the aldoximes, which is one of the reasons why

4 commercial extractants are often mixtures of aldoximes and ketoximes, although aldoximes are

5 known to be stronger copper complexants.

6 In this work, the oximation behavior of two selected carbonyl compounds in a liquid-liquid

7 reaction medium are studied. The main objective is to gain fundamental knowledge on the

8 oximation kinetics of salicylaldehyde and 2'-hydroxyacetophenone in a liquid-liquid reaction

9 system. The possibility to intensify the oximation reaction process by employing a flow reactor

10 with short contact time, but efficient mixing, was investigated. The selection of the above

11 carbonyl reagents was based on their similarity to the precursors of modern, industrially used,

12 hydroxyoxime liquid-liquid extractions reagents, such as 5-nonylsalicylaldoxime and 2-hydroxy-

13 5-nonylacetophenone oxime [1]. Degradation of hydroxyoximes under typical copper stripping

14 conditions was also briefly studied for both salicylaldoxime and 2'-hydroxyacetophenone oxime

15 to demonstrate the reversibility of the studied reaction. The findings from this study support the

16 development of on-site regeneration processes for the degraded hydroxyoxime organic ligands.

\section{EXPERIMENTAL}

\section{Reagents}

19 The carbonyl reagents, salicylaldehyde and 2'-hydroxyacetophenone, were purchased from

20 Sigma-Aldrich as analytical grade chemicals. Toluene (technical grade) was used as an organic

21 phase solvent. Hydroxylamine sulfate $\left(\mathrm{NH}_{3} \mathrm{OH}\right)_{2} \mathrm{SO}_{4}$ and $\mathrm{Na}_{2} \mathrm{CO}_{3}$ were also of analytical grade

22 (Sigma-Aldrich). Water purified with Centra 60/120 equipment was used in preparation of 23 aqueous solutions. 


\section{Analytical method}

2 The organic phases were analyzed by gas chromatography (GC) using a non-derivatized method

3 for determination of the carbonyl and oxime mole fractions. A gas chromatograph, Agilent

$46890 \mathrm{~N}$, with $25 \mathrm{~m}$ column, flame ionization detection (FID) and helium as a carrier gas was used

5 in the analysis. In the optimized method, the injector and detector temperatures were maintained

6 at $260{ }^{\circ} \mathrm{C}$ and $320^{\circ} \mathrm{C}$ respectively. The temperature of the column was adjusted according to the

7 following program: temperature was increased from $50{ }^{\circ} \mathrm{C}$ to $90{ }^{\circ} \mathrm{C}$ at $10^{\circ} \mathrm{C} / \mathrm{min}$ rate and after

8 that held constant for $2 \mathrm{~min}$. Thereafter it was ramped to $320^{\circ} \mathrm{C}$ at $10^{\circ} \mathrm{C} / \mathrm{min}$ rate, and held

9 constant for $2 \mathrm{~min}$ to give a total analysis time of $31 \mathrm{~min}$. Identification of components was done

10 with the help of external standard samples.

\section{Experiments in a continuous flow reactor}

12 Solutions of approximately $0.8 \mathrm{M}$ salicylaldehyde in toluene and $0.7 \mathrm{M}$ 2'-hydroxyacetophenone

13 (accurate concentration measured by GC) in toluene were prepared and used as stock solutions

14 for the organic phases. The aqueous phase stock solution was $1.7 \mathrm{M}\left(\mathrm{NH}_{3} \mathrm{OH}\right)_{2} \mathrm{SO}_{4}$, which

15 corresponds to $3.4 \mathrm{M}$ hydroxylamine. The $\mathrm{pH}$ of the aqueous solution was adjusted to 6.8 with

$16 \mathrm{Na}_{2} \mathrm{CO}_{3}$. The experiments were done in a $2.5 \mathrm{~mL}$ continuous stirred tank-reactor (CSTR)

17 immersed in a water bath and equilibrated to desired temperatures $\left(20-50{ }^{\circ} \mathrm{C}\right)$. Feed solutions

18 were warmed to the corresponding temperatures and pumped through the reactor by use of a

19 peristaltic pump (Ismatec JPN-12 or IPC). Mass flow rates of feed solutions were monitored with

20 the help of balances. Vigorous mixing in the reactor was achieved by using T8 Ultra Turrax high

21 shear mixer (by IKA) at $15000 \mathrm{rpm}$.

22 During the runs, the aqueous and organic streams were combined in a T-connector just before the 23 reactor. The exact $\mathrm{O} / \mathrm{A}$ volume ratio was calculated from mass flow data with measured densities 
1 of the aqueous and organic phases. The space time in the reactor, and the reaction temperature,

2 were varied. The total volumetric flow rate through the reactor was in the range of 1.6-10 $3 \mathrm{~mL} / \mathrm{min}$. Under steady state conditions, the extent of the oximation reaction was determined 4 offline through sampling of the dispersion from the reactor outlet. The phases in the samples 5 were immediately separated with a centrifuge (CINC V-2).

\section{Experiments in a batch reactor}

7 In batch experiments, the organic phase comprised of $0.84 \mathrm{M}$ of the aldehyde or $0.73 \mathrm{M}$ of the

8 ketone in toluene, and the aqueous phase was $1.7 \mathrm{M}\left(\mathrm{NH}_{3} \mathrm{OH}\right)_{2} \mathrm{SO}_{4}$ at $\mathrm{pH}$ 6.8. For each 9 experiment, $350 \mathrm{~mL}$ of aqueous phase was charged into a one-liter reactor and the temperature

10 was adjusted to the desired value. An equivalent volume of the organic phase was added to 11 obtain a phase ratio $\mathrm{O} / \mathrm{A}=1$. The content of the reactor was then stirred at 500-900 rpm for an 12 hour at a constant temperature. Samples of the dispersion were collected periodically, and the 13 phases in each sample were separated immediately by using a centrifuge to stop the reaction, and

14 to recover the oximated organic solution. The organic phase was analyzed for oxime and 15 carbonyl groups with the GC method described above.

\section{Hydrolytic degradation experiments}

17 Oximated organic solutions obtained after batch oximation of salicylaldehyde and 18 2'-hydroxyacetophenone were used as the organic phase for the degradation experiments. Two

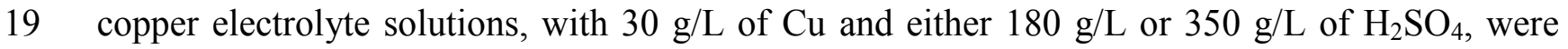
20 used. The total volume of the organic phase in each experiment was $400 \mathrm{~mL}$ with phase volume 21 ratio $\mathrm{O} / \mathrm{A}=1$. The experiments were conducted in $500 \mathrm{~mL}$ glass bottles at $40{ }^{\circ} \mathrm{C}$ in an orbital 22 shaker for one week. Samples were taken periodically and the recovered organic phases were 23 analyzed for the carbonyl and the oxime functional groups with GC as described above. 


\section{THEORY AND MATHEMATICAL MODELLING}

2 Oximation takes place in two steps, formation of a carbinolamine intermediate followed by its

3 dehydration, as illustrated in reaction Scheme I. The reaction mechanism for the carbinolamine

4 intermediate formation involves either acid catalyzed amine addition to carbonyl group or a non-

5 catalyzed formation of a zwitterion intermediate, which is converted to a neutral carbinolamine

6 complex after a proton switch either by water or acid catalyzed route [19-21]. Carbinolamine

7 formation rate is known to be influenced by $\mathrm{pH}$ and the structural reactivity of the carbonyl and

8 the amine compounds $[11-12,20,22]$. The decomposition of the intermediate into the oxime

9 occurs via acid [11] or base catalyzed dehydration [22-25].

10 The dehydration of carbinolamine proceeds rapidly both at low $\mathrm{pH}(\leq 2.5)$ and at high $\mathrm{pH}(\geq 10)$

11 [25] but is slow under neutral and mildly alkaline conditions [12, 20]. For example, the

12 formation of carbinolamine intermediate was reportedly observed in a homogeneous system

13 during oximation of acetone at $\mathrm{pH} 8.5$ but not at $\mathrm{pH} 2.5$ or at $\mathrm{pH} 10$ [25]. Dehydration of the

14 carbinolamine intermediate is considered the rate-determining step under neutral and mildly

15 basic reaction conditions [19]. In a more acidic or alkaline environment, uncatalyzed addition of

16 free base hydroxylamine to the carbonyl group is reported to be the rate-determining step [19-21].

17 It is also important to note that at low $\mathrm{pH}$ conditions, hydroxylamine exists mainly in protonated

18 form, which is more stable and less reactive to carbonyl compounds. In neutral and alkaline

19 conditions, most of the hydroxylamine is present in a more reactive free base form, which is

20 susceptible to decomposition even at ambient conditions [26].

21 According to Janakiraman and Sharma, and Fitzpatrick and Gettler $[4,10]$ the overall oximation

22 reaction is a second order reaction, being first order with respect to both the carbonyl group and

23 hydroxylamine. Shenk [14] suggests a third order or a pseudo-second order reaction including 
1 the role of the proton $(\mathrm{pH})$ in the oximation reaction mechanism. Fitzpatrick and Gettler [10] also

2 concluded, based on their oximation experiments with 11 carbonyl compounds, that the reaction

3 is irreversible under neutral $\mathrm{pH}$ conditions. However, as discussed already in the introduction, it

4 is known that the hydroxyoxime reagents used in the hydrometallurgical copper processes

5 undergo hydrolytic degradation under very acidic conditions. The mechanism for this reaction

6 has been proposed by Hurtado-Guzmán and Menacho [16] and presented in Scheme II.

\section{CSTR model}

8 The continuous reactor was modeled as an ideal CSTR at steady state with two immiscible liquid

9 phases as the feed. This is well justified based on the analysis of residence time distribution data

10 reported earlier [27] from the same high-shear mixer reactor. As will be explained below, only

11 the aldehyde oximation was studied in the CSTR. The mass balances of the reactants A

12 (salicylaldehyde) and HA (hydroxylamine) over the reactor are

$$
\dot{V}^{\mathrm{aq}}\left(c_{\mathrm{HA}}^{\mathrm{aq}, \text { feed }}-c_{\mathrm{HA}}^{\mathrm{aq}}\right)+r_{\mathrm{HA}} a_{\mathrm{int}} V_{\mathrm{R}}=0
$$

$$
\dot{V}^{\text {org }}\left(c_{\mathrm{A}}^{\text {org,feed }}-c_{\mathrm{A}}^{\text {org }}\right)+r_{\mathrm{A}} a_{\text {int }} V_{\mathrm{R}}=0
$$

16 In Eqs. (1) and (2), $c^{\mathrm{aq}}$ and $c^{\text {org }}$ are volumetric concentrations (mol/L) in the bulk of the two

17 phases, $V_{\mathrm{R}}$ is the volume (L) of the dispersion in the reactor and $a_{\text {int }}$ is the specific interfacial

18 area $\left(\mathrm{m}^{2} / \mathrm{L}\right)$. The volumetric flow rates of the aqueous and organic streams, $\dot{V}(\mathrm{~L} / \mathrm{s})$, give space

19 time $\tau=V_{\mathrm{R}}\left(\dot{V}_{\text {aq }}+\dot{V}_{\text {org }}\right)^{-1}$. Due to vigorous mixing in the high shear mixer, the space time is equal

20 for the two phases and the organic-to-aqueous phase ratio is $\phi=\dot{V}_{\text {org }} / \dot{V}_{\text {aq }}$. 
1 The interfacial area is not known and depends in practice on the droplet size distribution and the

2 phase ratio. Considering that the mixing rate was held constant in the CSTR experiments, and

3 that the phase ratio varied over a narrow range only $(\phi=0.92 \ldots 1.08)$, the droplet size

4 distribution is assumed to be independent of phase ratio. The interfacial area is thus related to the 5 phase ratio as

6

$$
a=\delta \frac{\phi}{1+\phi}
$$

7 where $\delta$ is an unknown constant characterizing the droplet size distribution. This constant also 8 scales the unit of $a$ from $1 / \mathrm{m}$ to $\mathrm{m}^{2} / \mathrm{L}$.

10 The rates of formation of $\mathrm{A}$ and $\mathrm{HA}$, denoted by $r\left(\mathrm{~mol} / \mathrm{m}^{2} / \mathrm{L}\right)$ in the mass balances, are 11 calculated by assuming that the net reaction at the interface is of first order with respect to both 12 reactants $[4,10]$

$$
r_{\mathrm{A}}=r_{\mathrm{HA}}=-k_{1}(T) q_{\mathrm{A}} q_{\mathrm{HA}}
$$

14 where $k_{1}$ is a reaction rate constant $\left(\mathrm{m}^{2} / \mathrm{mol} / \mathrm{s}\right)$ and $q$ denotes the interfacial concentrations $15\left(\mathrm{~mol} / \mathrm{m}^{2}\right)$ of the reactants.

17 Considering the high concentration of reactants in most of the experiments, hydroxylamine in 18 particular, the distribution of the reactants between the interface and the bulk phases can be non19 linear. In order to account for this phenomenon, the concentrations $q$ in Eq. (4) are calculated 20 with a simple model 


$$
q_{j}=\frac{q_{\mathrm{max}, j} b_{j} c_{j}^{k}}{1+b_{\mathrm{A}} c_{\mathrm{A}}^{\mathrm{org}}+b_{\mathrm{HA}} c_{\mathrm{HA}}^{\mathrm{aq}}}
$$

2 where $b$ and $q_{\max }$ are constants and superscript $k$ refers to the aqueous phase for $j=\mathrm{HA}$ and to the

3 organic phase for $j=$ A. Note that Eq. (5) is used here as an empirical model and not as a

4 thermodynamically consistent interfacial adsorption model. The presence of water and toluene at

5 the interface are not considered and the parameters $b$ and $q_{\max }$ are regarded independent of

6 temperature for simplicity.

8 In order to reduce the number of parameters, we define the interfacial rate of formation per

9 volume of the dispersion, $r_{j}^{\prime}(\mathrm{mol} / \mathrm{L} / \mathrm{s})$, as

11 which simplifies to

$$
r_{j}^{\prime}=r_{j} a_{\mathrm{int}}=-k_{1}(T) q_{\mathrm{max}, \mathrm{A}} q_{\mathrm{max}, \mathrm{HA}} \delta \frac{b_{\mathrm{A}} b_{\mathrm{HA}} c_{\mathrm{A}}^{\mathrm{org}} c_{\mathrm{HA}}^{\mathrm{aq}}}{1+b_{\mathrm{A}} c_{\mathrm{A}}^{\text {org }}+b_{\mathrm{HA}} c_{\mathrm{HA}}^{\mathrm{aq}}} \frac{\phi}{1+\phi}
$$

13 where $k_{1}^{\prime}$ is a constant that depends only on temperature according to an Arrhenius type

14 expression

$$
r_{j}^{\prime}=-k_{1}^{\prime}(T) \frac{c_{\mathrm{A}}^{\mathrm{org}} c_{\mathrm{HA}}^{\mathrm{aq}}}{1+b_{\mathrm{A}} c_{\mathrm{A}}^{\text {org }}+b_{\mathrm{HA}} c_{\mathrm{HA}}^{\mathrm{aq}}} \frac{\phi}{1+\phi}
$$


2 The mass balances were rewritten as in Eqs. (9) and (10) and the concentrations of A and HA in

3 their respective phases at the reactor outlet were solved numerically by using Eqs. (7) and (8) to 4 calculate the rates of formation.

$$
c_{\mathrm{HA}}^{\mathrm{aq}, \mathrm{feed}}-c_{\mathrm{HA}}^{\mathrm{aq}}+r_{\mathrm{HA}}^{\prime} \tau(1+1 / \phi)=0
$$

$$
c_{\mathrm{A}}^{\text {org,feed }}-c_{\mathrm{A}}^{\text {org }}+r_{\mathrm{A}}^{\prime} \tau(1+\phi)=0
$$

Batch reactor model

9 The concentrations of the reactants in their respective phases in the batch reactor were calculated

10 from the mass balances

$11 \frac{\mathrm{d} c_{\mathrm{HA}}^{\mathrm{aq}}}{\mathrm{d} t}=r_{\mathrm{HA}}^{\prime}(1+\varphi)$

$$
\frac{\mathrm{d} c_{\mathrm{A}}^{\mathrm{org}}}{\mathrm{d} t}=r_{\mathrm{A}}^{\prime}(1+1 / \varphi)
$$

13 where the phase ratio is $\varphi=V_{\text {org }} / V_{\text {aq }}$. The interfacial reaction rates were evaluated by using Eqs.

14 (7) and (8).

\section{Parameter estimation}

17 The four parameters of the kinetic model $\left(k_{\mathrm{ref}}, E_{\mathrm{a}}, b_{\mathrm{A}}, b_{\mathrm{HA}}\right)$ were fitted against $N=120 \mathrm{CSTR}$

18 runs at three temperatures and five aldehyde to hydroxylamine concentration ratios by

19 minimizing the sum of weighted squared residuals shown in Eq. (13) 


$$
R S S=\sum_{k=1}^{N} \omega_{k}\left(c_{\mathrm{A}, \mathrm{exp}, k}^{\mathrm{org}}-c_{\mathrm{A}, \mathrm{cal}, k}^{\mathrm{org}}\right)^{2}
$$

2 Owing to the wide range of concentrations (one decade in both hydroxylamine and

3 salicylaldehyde concentrations) the responses at low concentration were given a higher weight in

4 the parameter estimation by setting $\omega_{\mathrm{k}}=1 / c_{\mathrm{A}, \mathrm{exp}, \mathrm{k}}^{\mathrm{org}}$.

5 The oximation reaction rate constant in the batch reactor was estimated from data obtained with

$6500 \mathrm{rpm}$ and $900 \mathrm{rpm}$ stirring rates separately. Although the batch reactor runs were carried out

7 at $323 \mathrm{~K}$ only, the value of $k_{\mathrm{ref}}(\mathrm{T}=313 \mathrm{~K})$ was estimated instead of the value of $k_{1}^{\prime}(\mathrm{T}=323 \mathrm{~K})$ in

8 order to allow more direct comparison of the rate constants obtained in the two kinds of reactors.

9 Due to the limited amount of data, the values estimated from the CSTR experiments were used 10 for $E_{\mathrm{a}}, b_{\mathrm{A}}$ and $b_{\mathrm{HA}}$.

\section{RESULTS AND DISCUSSION}

\section{Batch reactor experiments: conversion and reversibility of the oximation reaction}

14 The rates of the forward and reverse reactions of the oximation reaction (see Scheme I and II)

15 depend strongly on $\mathrm{pH}$. In order to justify the assumption of irreversible oximation reaction at

16 the reaction conditions employed here $(\mathrm{pH}=6.8)$, batch reactor experiments were carried out

17 with carbonyl:hydroxylamine molar ratio of ca. 1:4. As observed in Fig. 1, the conversion of 18 salicylaldehyde to salicylaldoxime is practically complete at $20 \mathrm{~min}$. As the excess of 19 hydroxylamine was not very large, this result confirms the assumption in Scheme I that liquid20 liquid oximation of salicylaldehyde in toluene/water system can be treated as an irreversible 
1 reaction at neutral $\mathrm{pH}$ range of the aqueous phase. Considering the low amount of protons

2 present at this $\mathrm{pH}$ means that the reaction proceeds with the non-catalytic route.

3 The reverse reaction occurs in concentrated sulfuric acid, as is observed in Fig. 1B. The organic

4 phase was the toluene solution obtained in the oximation reaction experiments and the aqueous

5 phase corresponded to a typical stripping stage of hydrometallurgical copper processing, bearing

$630 \mathrm{~g} / \mathrm{L}$ of copper as a sulfate and $180 \mathrm{~g} / \mathrm{L}$ of sulfuric acid. Hydrolytic degradation of

7 salicylaldoxime was not complete but reached equilibrium within a few hours (the first data point

8 is at $8 \mathrm{~h}$ ). The equilibrium conversion of the aldehyde degradation was 0.81 and did not change

9 when sulphuric acid concentration was increased to $300 \mathrm{~g} / \mathrm{L}$. Under these conditions, the

10 oximation reaction proceeds with the acid-catalyzed pathways in Scheme I.

11 The liquid-liquid reaction of 2'-hydroxyacetophenone with hydroxylamine was found to be very

12 slow at $323 \mathrm{~K}$ and also at $343 \mathrm{~K}$. This could stem from lower reactivity of the ketone group

13 compared to the aldehyde group or from its lower affinity to the liquid-liquid interface.

14 According to [28], the octanol-water partition coefficients of 4-hydroxybenzaldehyde and 4'-

15 hydroxyacetophenone, which are structurally very close to the compounds used here, are equal

$16\left(\log P_{\mathrm{O} / \mathrm{w}}=1.35\right)$. Therefore, it is most likely that the lower reactivity of the ketone is related to

17 steric factors and the electron-withdrawing property of the methyl substituent group.

18 Also the degradation of the ketoxime in concentrated acid is slower than that of the aldoxime

19 (Figs. 1B and C). However, the ketoxime is completely hydrolyzed after approximately $50 \mathrm{~h}$ in

20 the batch experiments. It is thus probable that in commercial metals extraction reagents, that are

21 often mixtures of aldoximes and ketoximes, the ketoxime is more stable but, when degraded, it is

22 more difficult to regenerate by carrying out the reoximation reaction. It is important to note that 
1 degradation of commercial hydroxyoxime reagents takes place slower than these model 2 compounds due to the long alkyl substitute groups that make them more hydrophobic [18].

\section{Kinetics of aldehyde oximation in a CSTR}

5 The reaction kinetics in liquid-liquid oximation of salicylaldehyde with hydroxylamine were

6 investigated in more detail using a CSTR with vigorous high-shear mixing. The experimental

7 results are displayed in Figs. 2 and 3. The feed concentrations of both reactants in their

8 respective phases were varied over a large concentration range. As observed in the experimental

9 data, the reaction is fast at $323 \mathrm{~K}$, yielding approximately $50 \%$ conversion with $100 \mathrm{~s}$ space time.

10 In the batch reactor, equal conversion required approximately $180 \mathrm{~s}$ with the higher stirring rate

11 (900 rpm). It is thus expected that the mass transfer resistances are practically eliminated in the

12 high-shear mixer operating at $15000 \mathrm{rpm}$, and the kinetic parameters correspond to the kinetics

13 of the interfacial reaction only.

14 Owing to the high mixing power in the CSTR, the temperature at reactor outlet was typically $1 \%$ $15-2 \%$ higher than the set point. This was attributed to mixing rather than reaction heat because 16 conversion is rather small and a similar temperature effect is observed with this setup also for 17 non-reactive systems. The space time was within $\pm 5 \%$ and the phase ratio within $\pm 8 \%$ of the set 18 points in the experiments. Accurate values were recorded for each experiment and used in the 19 parameter estimation. Therefore, Figs. 2 and 3 display the experimental data with filled circles 20 and calculated results in parameter estimation with open circles for each CSTR experiment. The 21 solid lines are simulation results showing the dependence of aldehyde concentration at reactor 22 outlet on space time for a fixed set of parameters $\left(T, \phi, c_{\mathrm{A}}\right.$ and $\left.c_{\mathrm{HA}}\right)$ that correspond to average of 23 the experimental conditions in each set of experiments. 
1 The parameters of the kinetics model in Eqs. (3) to (5) were estimated from the CSTR data as

2 described above and are given in Table 1 together with their $95 \%$ confidence intervals. The

3 residuals are shown in Fig. 4. Overall, the fit is good although the data at lowest aldehyde

4 concentration in the feed shows some bias in the relative residuals. This corroborates the

5 assumption in Eq. (3) that the reaction can be regarded irreversible at $\mathrm{pH} 6.8$ in the liquid-liquid

6 system and the kinetics modelled using interfacial concentrations.

7 The activation energy was found to be relatively low, $34.2 \mathrm{~kJ} / \mathrm{mol}$, but not in the range of mass

8 transfer limited system. The reaction rate constant from the CSTR data has a relatively large

9 confidence interval. This is due to scatter in the data at high space time values and the fact that

10 the experimental concentration range is quite large. The $\beta$ parameters describe the distribution of

11 the aldehyde and the amine between bulk phases and the phase boundary where the reaction

12 occurs. The values should be interpreted such that the interphase has a higher A:HA mole ratio

13 than the overall mixture. The fact that the parameters did not tend towards zero during fitting

14 justifies the use of a simple correlation that cannot predict higher concentrations at the interface

15 than in the bulk phases. Excluding the distribution of reactants between the phases (i.e., using Eq.

16 (4) with $q_{\mathrm{A}}=c_{\mathrm{A}}^{\mathrm{org}} ; q_{\mathrm{HA}}=c_{\mathrm{HA}}^{\mathrm{aq}}$ ) lead to doubling of the norm of residuals from 0.115 to 0.218 . At

17 the same time, $k_{\text {ref }}$ decreased by a factor of $12 . E_{\text {a }}$ increased by only $9 \%$ indicating that the

18 distribution of solutes between the bulk and interface is not strongly dependent on temperature.

19 Reaction rate constants were estimated from the batch reactor data as well (Table 1) by using $b_{\mathrm{A}}$,

$20 b_{\mathrm{HA}}$, and $E_{\mathrm{a}}$ obtained from the CSTR data. The kinetics appears to be slower in the batch reactor.

21 This is as expected, since the mixing power with the high-shear mixer in the CSTR is

22 significantly higher than with a conventional turbine mixer used in the batch experiments. It is 


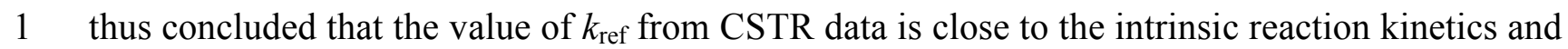
2 is not significantly affected by mass transfer resistance.

3

\section{CONCLUSIONS}

5 The results of this work show that oximation of salicylaldehyde with hydroxylamine in a

6 toluene-water liquid-liquid system follows an overall second order reaction kinetics at near

7 neutral $\mathrm{pH}$. It was shown that in the case of vigorous high-shear mixing in a CSTR, the reaction

8 kinetics is best modelled by correlating the distribution of the reactants between the bulk phases

9 and the phase boundary with a simple one-parameter (per reactant) model.

10 The kinetics of salicylaldehyde oximation was found to be much faster than that of 2'-

11 hydroxyacetophenone. Moreover, the degradation of the aldehyde, the backwards reaction, was 12 not complete in concentrated sulfuric acid / copper sulfate medium, typical for 13 hydrometallurgical processes, whereas the ketoxime was completely degraded in approximately

$1450 \mathrm{~h}$. The liquid-liquid system employed here seems suitable for use in industrial production of 15 aldoxime chemical reagents such as hydroxyaldoxime ligands applied in copper liquid-liquid 16 extraction.

\section{REFERENCES}

1. Szymanowski J, Hydroxyoximes and copper hydrometallurgy, Boca Raton USA: CRC press, pp.13-50 (1993).

2. Nagaraj DR and Somasundaran P, Chelating Agents as Collectors in Flotation: Oximes-Copper Minerals Systems. Mining Engineering 33:1351-1357 (1981). 
3. Finiels A and Geneste P, Micellar Catalysis in the Oximation Reaction of Aliphatic and Cyclanic Ketones. Hydrophobic Interactions. J. Org. Chem. 44 :2036-2038 (1978).

4. Janakiraman B and Sharma MM, Oximation of cycloalkanones (cyclododecanone and 4-tertbutylcyclohexanone): Micellar catalysis in slow and fast solid-liquid and liquid-liquid reaction systems. Chemical engineering science 40:223-233 (1985).

5. Osadchenko IM and Tomilov PA, Phase-Transfer Catalysis in Synthesis of Oximes. Russian Journal of Applied Chemistry 75:511-512 (2002).

6. Aakeröy BC and Sinha SA, Synthesis of ketoximes via a solvent-assisted and robust mechanochemical pathway. RSC advances 3:8168-8171 (2013).

7. Engel FJ and Chappelow CC, Preparation and properties of some hydroxy oximes and their precursors. Journal of Chemical \& Engineering Data 16:258-259 (1971).

8. Kopczyǹski T, Krzyżanowska E and Olszanowski A, Synthesis and Spectral Data of $(E)$ and (2) Oximes of 2-Hydroxybenzophenone Derivatives. Journal für Praktische Chemie 331:486-492 (1989).

9. Tyman HPJ and Iddenten JAS, The synthesis of oxime reagents from natural and semi-synthetic phenolic lipid and alkanoic acid resources for solvent recovery of copper(II). Journal of Chemical Technology and Biotechnology 80:1319-1328 (2005).

10. Fitzpatrick WF and Gettler DJ, Kinetics of Oxime Formation; Temperature Coefficients of Rate of Formation of Several Oximes. J. Am. Chem. Soc. 78:530-536 (1956).

11. Brighente IMC and Yunes RA, The general mechanisms of attack of nitrogen nucleophiles on carbonyl compounds. Facts that determine the change of rate-pH profiles. J. Braz. Chem. Soc. 8:549-553 (1997).

12. Calzadilla M, Malpica A and Cordova $T$, Effect of structure on reactivity in oxime formation of benzaldehydes. Journal of Pyhysical Organic Chemistry 12:708-712 (1999).

13. Córdova T, Peraza AJ, Calzadilla M and Malpica A, Kinetics and mechanism for oxime formation from methyl pyruvate, J.Phys. Org.Chem. 15:48-51 (2002).

14. Shenk HG, Organic functional group analysis: Theory and development, $1^{\text {st }}$ edtion, UK: Pragamon Press Ltd, pp.3-20 (1968). 
15. Whewell JR, Foakes JH and Hughes AH, Degradation in hydrodroxyoxime solvent extraction systems. Hydrometallurgy 7:7-26 (1981)

16. Hurtado-Guzman, C. and Menacho, J.M. Oxime degradation chemistry in copper solvent extraction plants. Copper 2003-Cobre 2003, Vol. VI, Book 2, Santiago, Chile, ed. Riveros PA, Dixion DG, Dreisinger DB and Menacho HJ, Hydrometallurgy of copper : Modelling, impurity control and solvent extractaion, Montreal, pp.719-734 (2003).

17. Paatero E, Virolainen S, Kortelainen $\mathrm{J}$ and Jyrkkä K, Regeneration of degraded Hydroxyoxime reagents, ed. Valenzuela L F. and A. Moyer A B, ISEC 2011, Santiago, pp.1-6 (2011).

18. Gang ZX and Qian Z, The stability of copper Extractants in Acid Media, ALTA 2014 NickelCobalt-Copper Proceedings, pp.281-291 (2014).

19. Reimann JE, Jencks PW, The mechanism of nitrone formation. A defense of anthropomorphic electrons. J Am Chem Soc. 88:3973-3982 (1966).

20. Sayer JM, Pinsky B, Schonbrunn A and Washtien W, Mechanism of carbinolamine formation. Journal of the American Chemical Society 96:7998-8009 (1974).

21. Sayer JM and Jencks WP, Mechanism and catalysis of 2-methyl-3-thiosemicarbazone formation. A second change in rate-determining step and evidence for a stepwise mechanism for proton transfer in a simple carbonyl addition reaction. Journal of the American Chemical Society 95: 5637-5649 (1973).

22. Rosenberg S, Silver SM, Sayer JM and Jencks WP, Evidence for two concurrent mechanisms and a kinetically significant proton transfer process in acid-catalyzed O-methyloxime formation. Journal of the American Chemical Society 96: 7986-7998 (1974).

23. Simionatto EL, Yunes PR and Yunes RA, The Effect of Boric Acid on the Dehydration Step in the Formation of Oxime from salicylaldehyde. J.Chem.Soc.Perkin Trans. 2:1291-1294 (1993)

24. Malpica A, Calzadilla M, Cordova TC, Torres S and Saulny GH, Kinetics and mechanism for oxime formation from 4-dimethylamino-benzaldehyde and 4-trimethylammonio-benzaldehyde iodide. International Journal of Chemical Kinetics 31:387-392 (1998).

25. Keglevich G, Csontos I, Szilágyi N and Greiner I, A Study of the pH Dependence of the Two-Step Oximation of Acetone by in situ Fourier Transform Infrared Spectroscopy. Chem. Eng. Technol 31:421-425 (2008). 
26. Cisneros LO, Rogers WJ and Mannan MS, Comparison of the Thermal Decomposition Behaviour for Members of the Hydroxylamine Family. Thermochimica Acta 414:177-183 (2002).

3 27. Tamminen, J., Sainio, T., Paatero, E., Intensification of metal extraction with high-shear mixing, Chem. Eng. Process. Process Intensif., 73: 119-128 (2013).

5 28. Golmohammadi, H., Prediction of octanol-water partition coefficients of organic compounds by multiple linear regression, partial least squares, and artificial neural network. J. Comput. Chem., 30: 2455-2465 (2009). 
1

2

\section{List of figure captions}

4 Figure 1.

5 Oximation of 2'-hydroxyacetophenone (squares) and salicylaldehyde (circles) with 6 hydroxylamine in a batch reactor at $\mathrm{pH} 6.8$ (A). Reaction temperature: $323 \mathrm{~K}$ (red, yellow) or $7343 \mathrm{~K}$ (blue). Stirring rate: $500 \mathrm{rpm}$ (green) or $900 \mathrm{rpm}$ (red, blue). Solid lines: calculated with 8 the batch reactor model. Initial concentrations: $0.73 \mathrm{M}$ for the ketone, $0.84 \mathrm{M}$ for the aldehyde 9 and $3.4 \mathrm{M}$ for hydroxylamine. Phase ratio = 1.0. Hydrolysis of the corresponding aldoxime $(\mathrm{B})$ 10 and ketoxime (C) in $30 \mathrm{~g} / \mathrm{L} \mathrm{CuSO}_{4}$ solutions with $180 \mathrm{~g} / \mathrm{L}$ (red circles) or $300 \mathrm{~g} / \mathrm{L}$ (crosses) $11 \mathrm{H}_{2} \mathrm{SO}_{4}$ at $313 \mathrm{~K}$. Phase ratio $=1.0$.

13 Figure 2.

14 Liquid-liquid oximation of salicylaldehyde (in toluene) and hydroxylamine (in water, $c_{\mathrm{HA}}^{\text {feed }}=3.4$

$15 \mathrm{M}, \mathrm{pH}$ 6.8) in CSTR. Filled yellow circles: experimental data. Open circles: calculated with

16 exact values of operating conditions $\left(T, \tau, \phi, c_{\mathrm{j}}\right)$ in each data point. Lines: calculated using 17 average values of operating conditions.

19 Figure 3.

20 Liquid-liquid oximation of salicylaldehyde (in toluene) and hydroxylamine (water, $\mathrm{pH}$ 6.8) in a

21 CSTR. Filled circles: experimental data; magenta: $c_{\mathrm{HA}}^{\text {feed }}=0.34 \mathrm{M}$, green: $c_{\mathrm{HA}}^{\text {feed }}=1.7 \mathrm{~mol} / \mathrm{L}$, red: 
$1 \quad c_{\mathrm{HA}}^{\text {feed }}=3.4 \mathrm{M}$. Open circles: calculated with exact values of operating conditions $\left(T, \tau, \phi, c_{\mathrm{j}}\right)$ in

2 each data point. Lines: calculated using average values of operating conditions.

$4 \quad$ Figure 4

5 Goodness of fit and residuals in estimation of kinetic parameters for two-phase oximation of

6 salicylaldehyde with hydroxylamine in a CSTR equipped with a high shear mixer. Reaction

7 temperatures: triangle $=293 \mathrm{~K}$, square $=313 \mathrm{~K}$, circle $=323 \mathrm{~K}$. Colors indicate the feed

8 concentration of hydroxylamine in the aqueous phase: green $=0.34 \mathrm{M}$, blue $=1.7 \mathrm{M}$, red $=3.4 \mathrm{M}$. 
Table 1. Parameters of the kinetic model in Eqs. (7) and (8) for oximation of salicylaldehyde (in toluene) with hydroxylamine (in water, $\mathrm{pH} 6.8$ ) and their $95 \%$ confidence intervals. $T_{\text {ref }}=313 \mathrm{~K}$.

\begin{tabular}{llll}
\hline Parameter & CSTR & Batch (500 rpm) & Batch (900 rpm) \\
\hline$k_{\text {ref }}\left(T_{\text {ref }}\right), \mathrm{L} / \mathrm{mol} / \mathrm{s}$ & $(10.55 \pm 4.8) \cdot 10^{-3}$ & $(5.82 \pm 0.4) \cdot 10^{-3}$ & $(6.58 \pm 0.6) \cdot 10^{-3}$ \\
$E_{\mathrm{a}}, \mathrm{kJ} / \mathrm{mol}$ & $34.7 \pm 2.6$ & - & - \\
$b_{\mathrm{A}}$ & $0.907 \pm 0.37$ & - & - \\
$b_{\mathrm{HA}}$ & $0.389 \pm 0.14$ & - & - \\
\hline
\end{tabular}




\section{Scheme I}
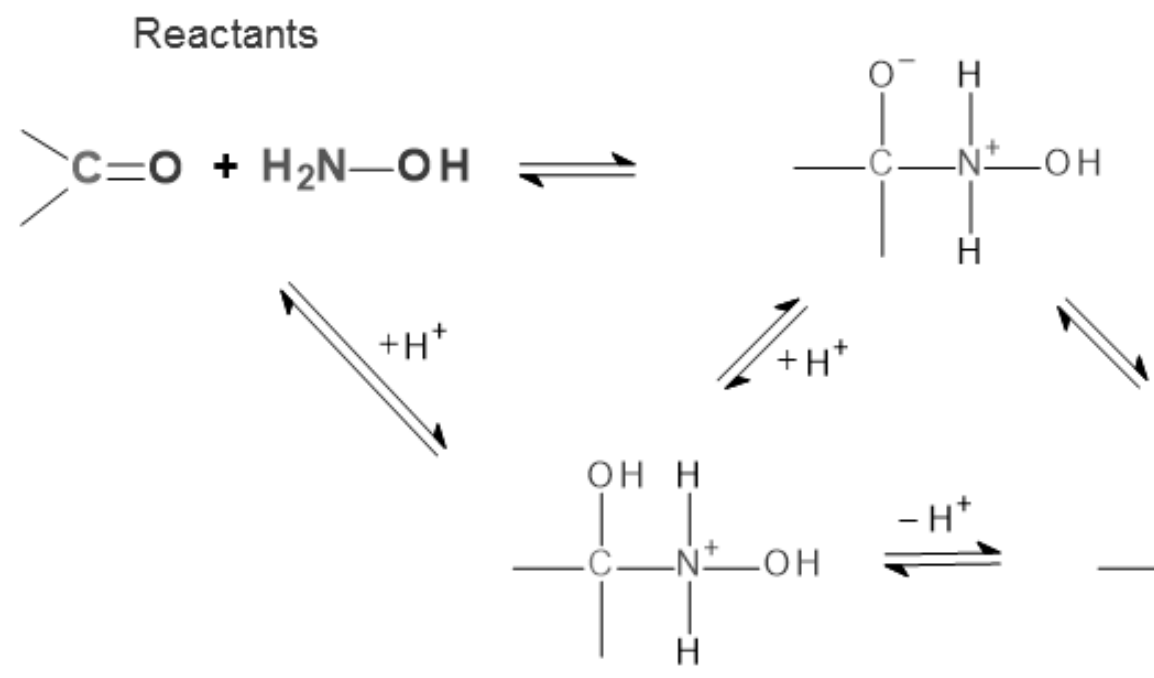<smiles>CC(C)(O)NO</smiles>

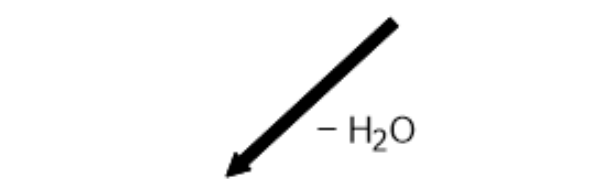

29

30

31

32

33

34

35

36

37

38

39

40

41

42

43

44

45

46

47

48

49

50

51

52

53

54

55

56

57

58

59

60

http://mc.manuscriptcentral.com/jctb-wiley 


1
2
3
4
5
6
7
8
9
10
11
12
13
14
15
16
17
18
19
20
21
22
23
24
25
26
27
28
29
30
31
32
33
34
35
36
37
38
39
40
41
42
43
40
45
49
50
51
52
53
55
50

\section{Scheme II}

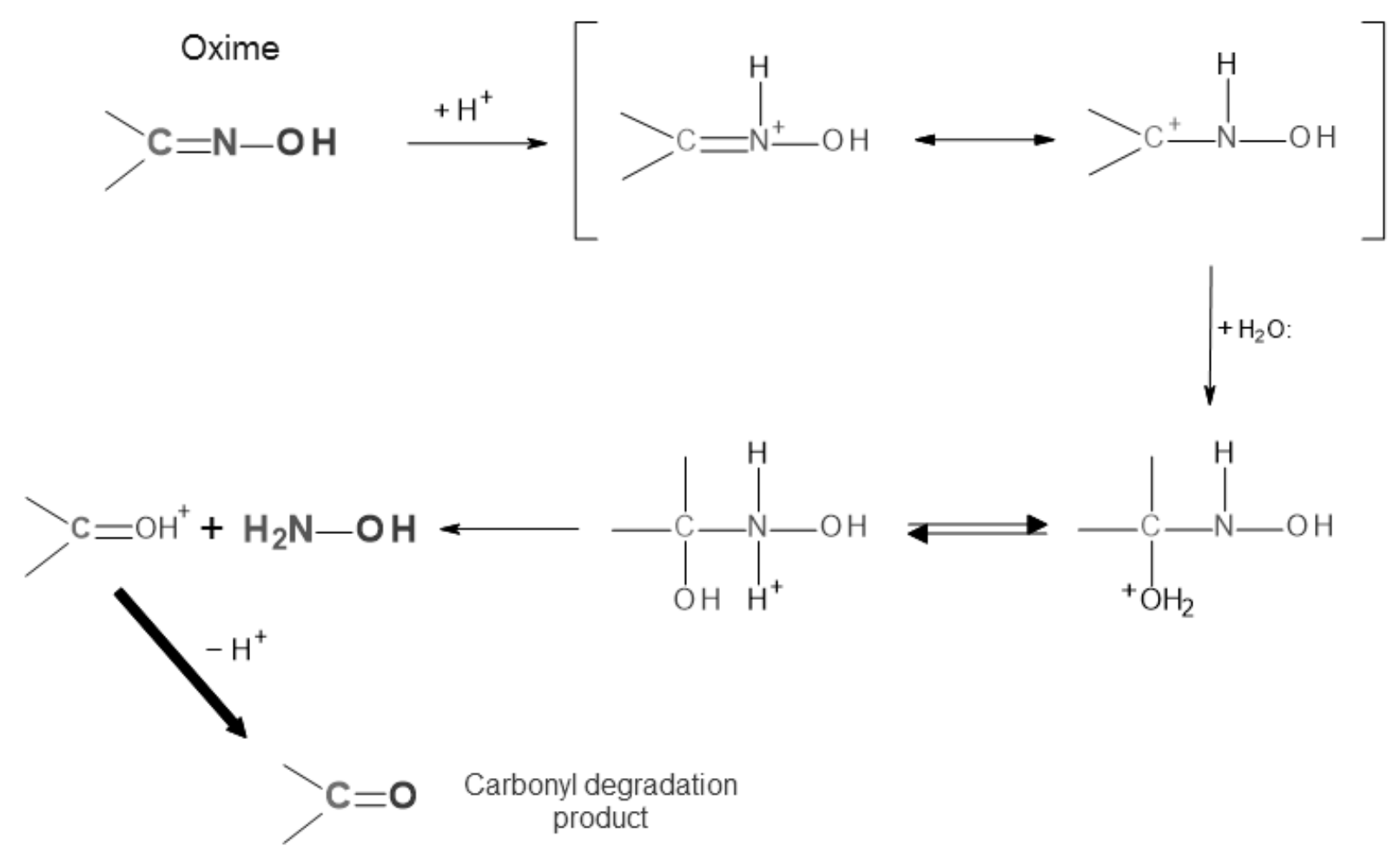


A)

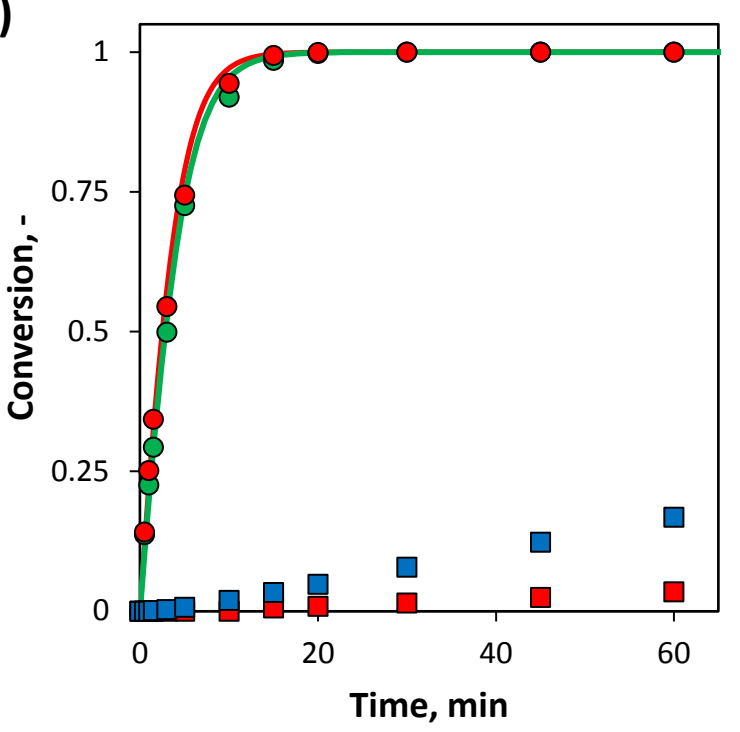

B)

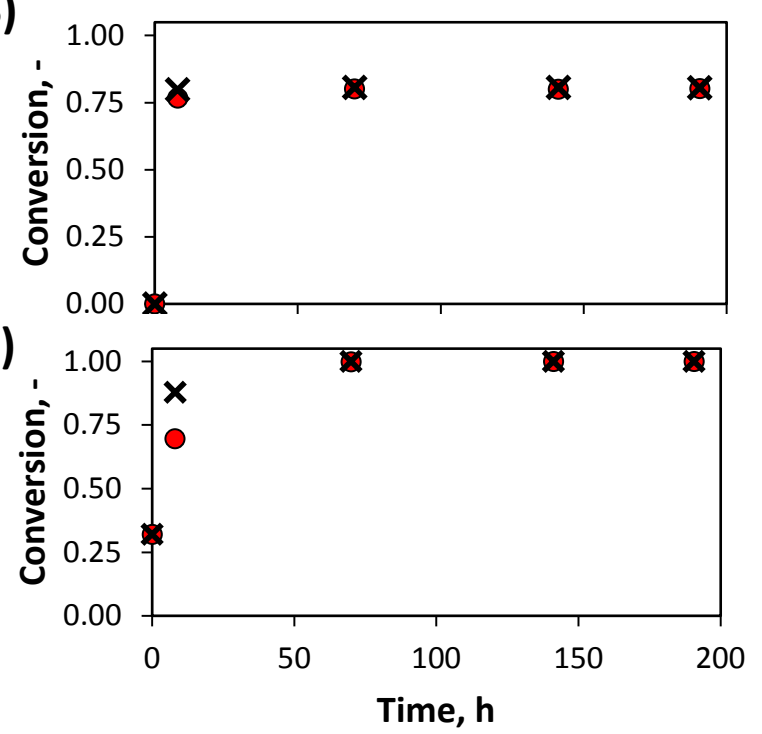

Figure 1. 
1

2

3

4

5

6

7

8

9

10

11

12

13

14

15

16

17

18

19

20

21

22

23

24

25

26

27

28

29

30

31

32

33

34

35

36

37

38

39

40

41

42

43

44

45

46

47

48

49

50

51

52

53

54

55

56

57

58

59

60
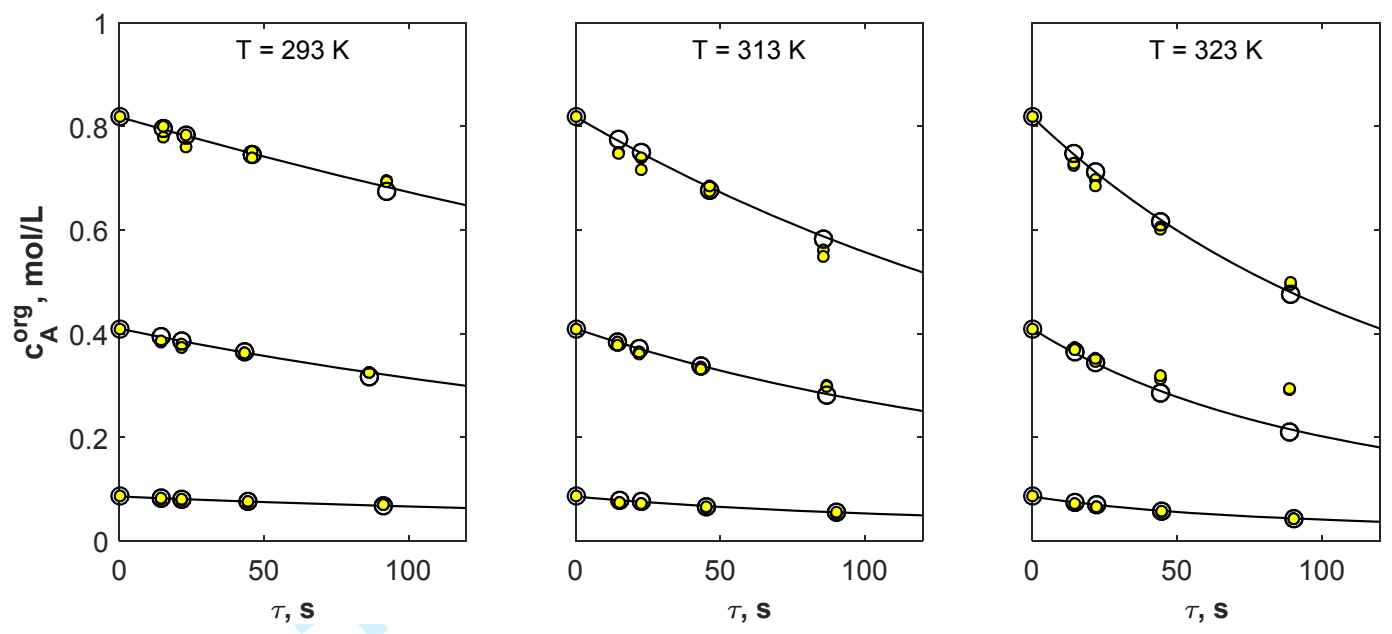

Figure 2. 

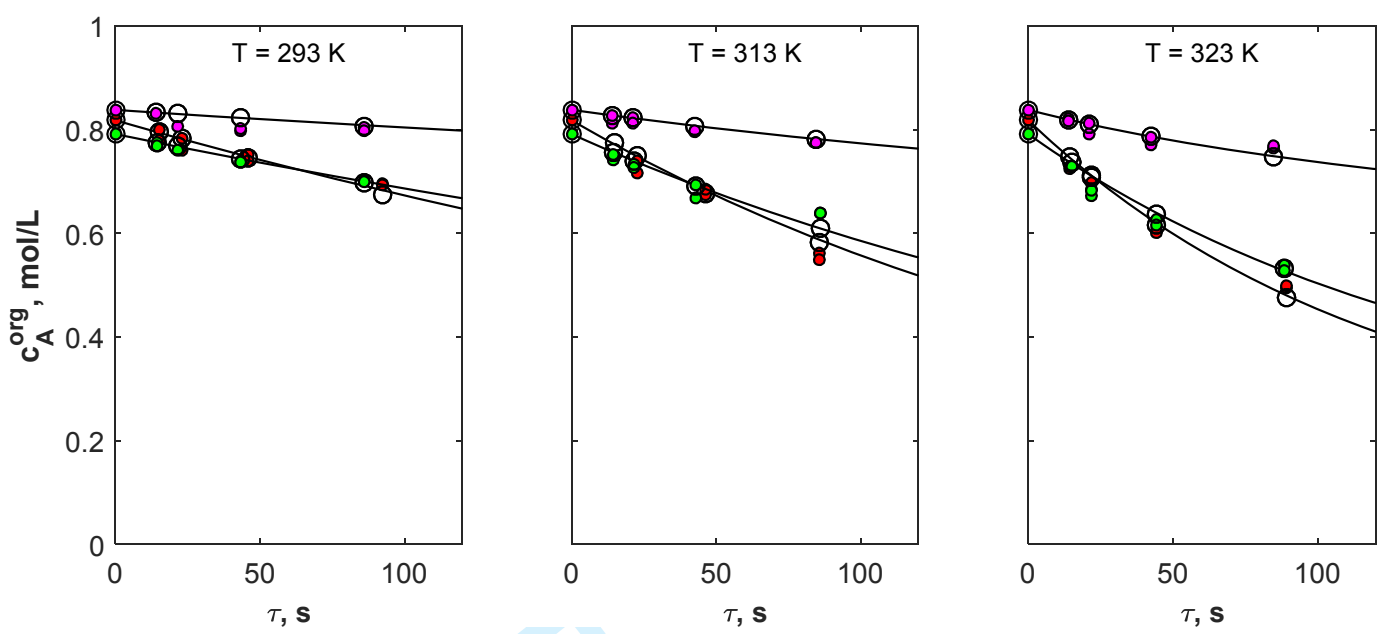

Figure 3.

http://mc.manuscriptcentral.com/jctb-wiley 

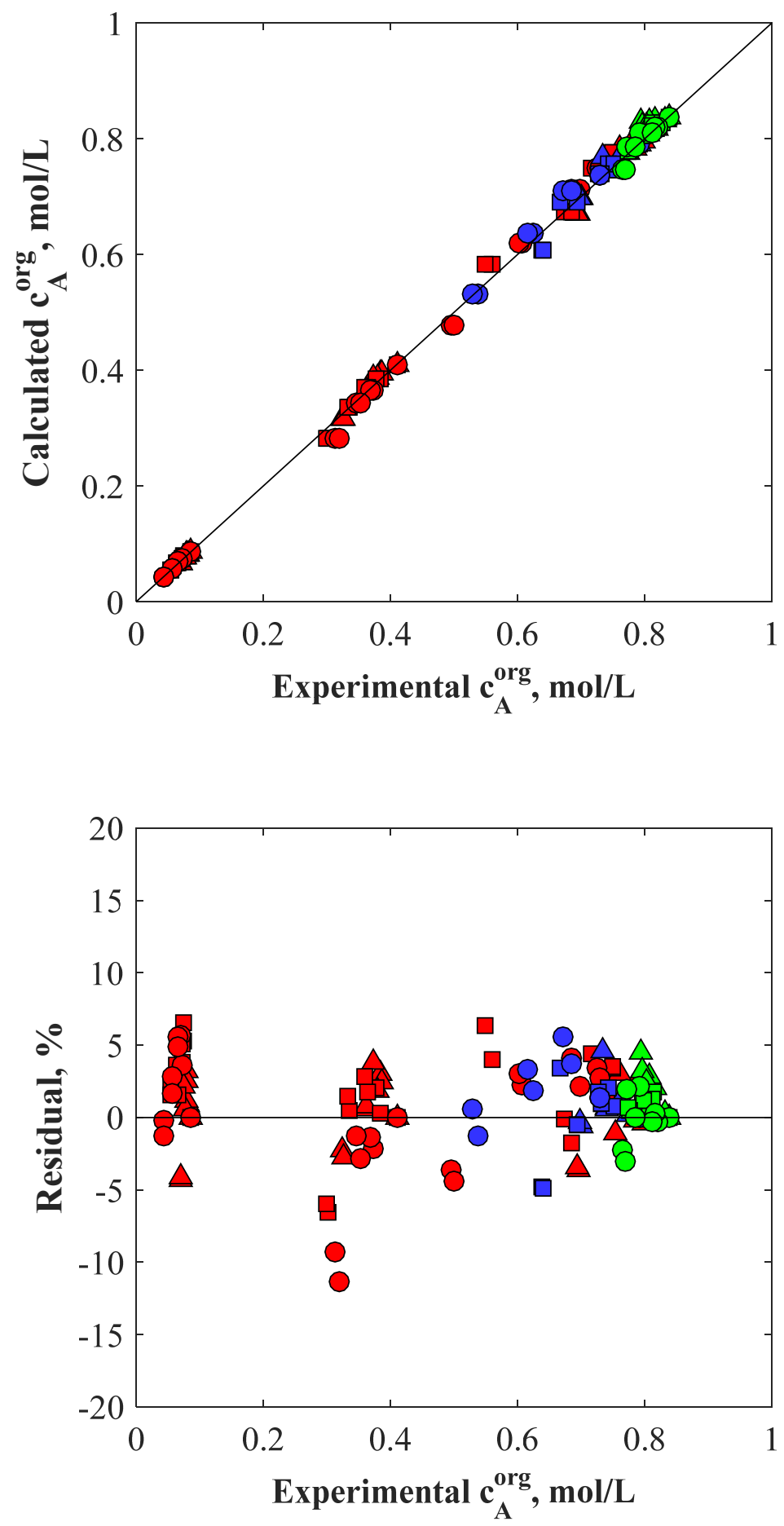

Figure 4. 